\title{
Real-time image processing systems using fuzzy and rough sets techniques
}

\author{
Gwanggil Jeon ${ }^{1} \cdot$ Marco Anisetti ${ }^{2} \cdot$ Ernesto Damiani $^{3} \cdot$ Olivier Monga ${ }^{4}$
}

Published online: 6 January 2018

c) Springer-Verlag GmbH Germany, part of Springer Nature 2018

Multimedia systems are increasingly required to provide advanced real-time features with reliable performance and low cost. In particular, real-time image processing techniques are used in a number of domains of science, technology, and business. Emerging requirements for such techniques include fast detection and exploitation of any valuable information that can be extracted from multimedia content, even in presence of uncertainty and noise. Although some basic building blocks of real-time image processing are already in place, many challenging issues remain to be resolved. In this special issue, we focus on how extract valuable information in real time from images which are incomplete, noisy, imprecise, fragmentary, vague, contradictory, deficient, and overloaded, and refine it reliably within the short deadlines of practical multimedia applications. In other words, we look for contributions describing how uncertain data are transitioned to reliable information which are easily treated.

This special issue is intended to provide a highly recognized international forum to presenting innovative developments of fuzzy set applications in real-time image processing systems. The ultimate objective is to bring together wellfocused, top-quality research contributions, providing to the general image processing community an opportunity to get an overall view of recent results, to identify the most promising avenues and to promote the visibility and relevance of

Marco Anisetti

marco.anisetti@unimi.it

Gwanggil Jeon

gjeon@inu.ac.kr

Ernesto Damiani

ernesto.damiani@kustar.ac.ae

Olivier Monga

olivier.monga@ird.fr

1 Incheon National University, Incheon, Korea

2 Università degli studi di Milano, Milan, Italy

3 EBTIC, Khalifa University, Abu Dhabi, UAE

4 Institut de Recherche pour le Developpement, Marseille, France fuzzy techniques. The intent is to raise collective awareness of the domain of real-time image processing technologies as a highly promising area to be pursued by the fuzzy and rough set research community.

Exploring sparse representation to enhance the resolution of infrared image has attracted much attention in the last decade. However, conventional sparse representation-based super-resolution aim at learning a universal and efficient dictionary pair for image representation. The contribution by $\mathrm{Wu}$ et al. "Multiple dictionary pairs learning and sparse representation-based infrared image super-resolution with improved fuzzy clustering" proposes an improved fuzzy clustering and weighted scheme reconstruction framework to solve this problem. Firstly, the training patches are divided into multiple clusters by joint learning multiple dictionary pairs with improved fuzzy clustering method. Then, highresolution patches are estimated according to several most accurate dictionary pairs. Finally, these estimated highresolution patches are integrated together to generate a final HR patch by a weighted scheme. Numerous experiments demonstrate that this framework outperforms some state-ofthe-art super-resolution methods in both quantitatively and perceptually.

Clear images are critical in understanding real scenarios. However, the quality of images may be severely declined due to terrible conditions. Images exposed to such conditions are usually low contrast, contain much noise, and suffer from weak details. The contribution by Yang et al. "Retinex-based image enhancement framework by using region covariance filter" proposes a retinex-based image enhancement framework that can increase contrast, eliminate noise, and enhance details at the same time. There are four steps in this work: (1) utilize a region covariance filter to estimate the illumination accurately at multiple scales; (2) utilize contrast-limited adaptive histogram equalization to enhance the global contrast of original images; (3) adopt a non-local means filter to eliminate noise and use a guided filter to enhance the details in the reflectance; (4) synthesize the final enhanced image by fusing the enhanced illumination and reflectance at each 
scale. Simulations show the improvement of the proposed framework in terms of both visual perception and quantitative comparisons with other compared methods.

The ramp entrance connects the main road and the ramp and is the merging zone for vehicles from main road and ramp. The contribution by Cui et al. "Early ramp warning using vehicle behavior analysis" focuses on the early warning to avoid collision of vehicles for the ramp entrance on freeway. Firstly, authors studied the relation between the vehicle speed and collision risk by analyzing the vehicle trajectories. Secondly, the risk grades were defined based on the lag of the time when vehicles enter into the merging zone from the main road and the ramp. Finally, the risk grade was released by the information board and vehicle terminal to make driver aware of it, so that the vehicles can pass the merging zone in sequence rather than collide with each other. Theoretical analysis and experiment results show that the proposed method could realize early warning for the ramp entrance, reducing the possible traffic accidents.

The vehicle behavior analysis is often based on the motion trajectory analysis, which lays the foundation for many applications such as velocity detection, vehicle classification, and vehicle counting. In the contribution by Wang et al. "Vehicle trajectory clustering based on 3D information via a coarse-to-fine strategy," a trajectory clustering framework is proposed for vehicle trajectory analysis. Firstly, feature points are extracted by the algorithm of Oriented FAST and Rotated BRIEF (ORB) which uses binary strings as an efficient feature point descriptor. Secondly, a matching method based on Hamming distance is used to obtain the tracking trajectory points. Finally, a novel clustering method, which contains three phrases, i.e., coarse clustering, fine clustering, and agglomerative clustering, is proposed to classify vehicle trajectory points based on the 3D information in real traffic video. Experimental results demonstrate that the accuracy of the proposed method can reach $95 \%$.

Image noise can range from almost imperceptible specks on a digital photograph taken in good light, to optical and radioastronomical images that are almost entirely noise, from which a small amount of information can be derived by sophisticated processing. In the contribution by Kim et al. "Adaptive switching filter for impulse noise removal in digital content," a new fuzzy-rule-based impulse noise denoising method which removes unwanted artifacts and reconstructs original images is proposed. The proposed method is based on the fuzzy rule selective approach, which has four submethods. The proposed method can effectively remove noise artifacts that are caused by various levels of impulse noise. Experimental result show that the presented method outperforms conventional methods.

Due to factors such as snow and ice impeding drivers' vision, the number of automobile crashes significantly rises during winter months. The contribution by Bai et al. "Sensor- based risk perception ability network design for drivers in snow and ice environmental freeway: a deep learning and rough sets approach" studies on automatic evaluation network of the risk perceived ability for motorists driving on the freeway in snow and ice environments, using a deep learning approach and the rough sets technique. This approach can provide reference for the design of hazard detection systems of partially automated vehicles.

Medical imaging techniques play a very important role in modern life. However, due to the technique limitation, the random noise often degrades the quality of acquired medical images, which seriously affects the medical image analysis. The contribution by Bai et al. "Medical image denoising based on sparse dictionary learning and cluster ensemble" proposes a denoising scheme which combines sparse dictionary learning with cluster ensemble. In this work, the effective cluster ensemble method is utilized to gain the class label of image feature set. Moreover, an adaptive dictionary was trained by the sparse dictionary learning algorithm. Simulations validate the superiorities of the proposed method and have a satisfactory speed.

Recently, hyper-spectral image segmentation and classification has been a challenging and critical task in remote sensing applications. Although many techniques and algorithms were proposed, there are still some drawbacks, such as being data dependent, having decor relates among the frequency bands, requiring high storage, image quality being not so impressive and large in size. The contribution by Gopalan et al. "Segmentation and classification of hyperspectral images using local Horizontal Vertical pattern extraction grid" proposes a new system for hyper-spectral image segmentation and classification. At first, the hyper-spectral image is given as the input of preprocessing, where the unwanted noise is eliminated and the image is smoothened for further processing. Then, the patterns are extracted from the filtered image by using pattern extraction grid in which the circular local binary pattern and the sorted local Horizontal Vertical are used to extract the patterns.

Online weather forecasts and weather apps enable viewers to actively retrieve information. However, television weather forecasting uses a format, which allows viewers to unilaterally receive information from presenters speaking fast for a short period of time. In the contribution by Kim "Chameleonlike weather presenter costume composite format based on color fuzzy model," an alternative method of weather forecast is proposed, consisting of using the presenter's clothes as a tool for providing information by exploiting the reactions of viewers focusing on the presenter. Specifically, this study constructed the color fuzzy model to represent temperature, fine dust level, and humidity by hue, saturation, and value, respectively. The weather presenter's clothing transforms like a chameleon, in real time, according to content and provides emotional information to viewers. 
Vessel segmentation is a critical and challenging task for fundus image processing, which is precursor and essential first step to further vessel measurement and diagnosis. The contribution by Jiang et al. "Automatic vessel segmentation on fundus images using vessel filtering and fuzzy entropy," proposes a novel hybrid automatic vessel segmentation method for the delineation of vessels on fundus images. The method consists of two main steps including Hessianbased vessel filtering and vessel segmentation. In vessel filtering, multiscale linear filtering based on Hessian matrix is adapted to enhance vessels in the image. After vessel filtering, a novel two-dimensional histogram of filtering image is generated. Then the thresholds are determined by the fuzzy entropic concepts. Compared to conventional approaches, the proposed method yields more complete and accurate results.

In the contribution by Shi et al. "A memetic algorithm based on MOEA/D for the examination timetabling problem," a memetic algorithm based on MOEA/D is presented to deal with the uncapacitated multiobjective examination timetabling problem in this paper. The examination timetabling problem is considered as a two-objective optimization problem in this paper, while it is modeled as a single-objective optimization problem generally. The framework of a multiobjective evolutionary algorithm with decomposition (MOEA/D) is first employed to guide the evolutionary process. Two special local search operators are designed to find better individuals. Simulations prove that the proposed algorithm can produce a promising solutions for each instance.

Recently, the singular value decomposition (SVD) method has been used as preprocessing method in order to deal with high-dimensional data and achieve fuzzy-rough reduct convergence on higher dimensional datasets. Although it is a well-known fact that SVD offers attractive properties, its high computational cost remains a critical issue. The contribution by Cuomo et al. "On GPU-CUDA as preprocessing of fuzzy-rough data reduction by means of singular value decomposition," presents a parallel implementation of the SVD algorithm on graphics processing units using CUDA programming model. The proposed method is based on an iterative parallel version of the QR factorization by means of Givens rotations using the Sameh and Kuck scheme.

These days, city traffic is monitored by static network cameras deployed on few places of highways. Most of the vehicles are also equipped with cameras to store the videos as a black box. However, monitoring and controlling city traffic by using these thousands of cameras produce an overwhelming amount of high-speed videos, which is challenging to process at real time. The contribution by Paul et al. "Realtime video processing for traffic control in smart city using Hadoop ecosystem with GPUs," proposes a system to control city traffic by identifying illegal traffic behavior, such as illegal U turn, through continuous monitoring of city traffic. The continuous city traffic is monitored by the network static cameras placed on the road as well as by all the vehicles' cameras. Authors proposed architecture to process high-speed overwhelming amount of traffic videos at real time. Results show that the proposed system with GPUs and Hadoop ecosystem is more efficient as compared to existing CPU-based MapReduce implementation.

The contribution by Lee et al. "Real-time implementation of a robust active control algorithm for narrowband signals suppression," presents a practical active noise control (ANC) method with robust stability for reducing the powertrain noise or vibration inside a car. It is important to ensure that a practical ANC system for a car is robustly stable to variations or uncertainties in the actual plant. After investigating the robust stability condition of the ANC algorithm, a robust plant model is designed by considering the multiplicative plant uncertainties within given bounds such as closing or opening door windows. The ANC algorithm was implemented in a dSPACE DS1401 as a control platform, an error microphone and a subwoofer as a secondary source were positioned at the driver's left ear and the trunk of the experimental car, respectively. The real-time control experiments were carried out against the plant perturbation when the engine was either idling or sweeping in the neutral mode.

Transform-based techniques partially address challenges like robustness and the imperceptibility in image steganography. Such approaches, however, increase the memory requirement and reduce the quality of the cover image and hiding capacity. The contribution by Ahmad et al. "An adaptive hybrid fuzzy-wavelet approach for image steganography using bit reduction and pixel adjustment" presents an adaptive hybrid method for image steganography procedure based on bit reduction and pixel adjustment using the fuzzy logic and integer wavelet transform technique. The fuzzy set theory provides powerful tools to represent and process human knowledge in the form of fuzzy if-then rules that can resolve difficulties in image processing arising due to the uncertainty of the data, tasks, and results. Simulation results demonstrate the effectiveness of the proposed approach.

Personalized data collected from smartphones and similar devices reflect time dependency. Currently, research using such data as a basis for prediction of future data is ongoing. This suggests that not only is time dependency reflected in past data but also in newly produced data, and that time-dependent weights also are reflected therein. The contribution by Kang et al. "Outgoing call recommendation using neural network" analyzes the most prominent feature of personalized data, the call log. The random forest (RF) method was used to find highly correlated data between call data and new Outgoing Calls. This information was then learned through a Neural Network. "Recent call volume" and "changing data" showed a high correlation, and experiments were conducted so that time change could be adequately reflected. 
The problem of repetitions was addressed while maintaining the hit ratio.

The use of computer assisted decision systems (CAD) for the diagnosis of skin cancer dermoscopy is aggravated by the potential gains of its excellent performance. It automates the skin lesion analysis, and reduces the amount of repetitive and tedious tasks to be done by physicians. The contribution by Ahmad et al. "Melanocytic and nevus lesion detection from diseased dermoscopic images using fuzzy and wavelet techniques" is mainly focused on the computer vision perspective to design a CAD system which will facilitate the physicians. An automated PR system that includes four inter related processes to analyze of skin lesions by the clinicians: image preprocessing, segmentation, feature extraction, feature selection, and classification. This work proposes novel techniques for lesion detection and classification on peripheral part of the lesion using M-Medoid classier along with the contrast of patterns. Classification results obtained from the proposed feature matrix were compared with some other texture descriptors, showing the superiority of the proposed descriptor.

In the contribution by Wang et al. "Wavelet-contentadaptive BP neural network-based de-interlacing algorithm," authors introduce an intra-field deinterlacing algorithm based on a wavelet-content-adaptive back propagation (BP) neural network (BP-NN) using pixel classification. During interpolation, there is an issue of different image features having completely different properties, such as smooth regions, edges, and textures. Authors use the wavelet transform to divide the images into several pieces with different properties. Then, each piece has similar image features and each one is assigned to one neural network. The BP-NN-based deinterlacing algorithm can reduce blurring by recovering the missing pixels via a learning process. Compared with existing deinterlacing algorithms, the proposed algorithm improves the peak signal-to-noise ratio and visual quality while maintaining high efficiency.

In the contribution by Ashraf et al. "Stratified modeling in soft fuzzy topological structures," authors introduce the concepts of stratified fuzzy soft topogenous, stratified fuzzy soft filter, stratified fuzzy soft quasi-proximity and stratified fuzzy soft grill. They introduce the concept of fuzzy soft topogenous structures by combining fuzzy soft topogenous with fuzzy soft filter and introduce the concept of fuzzy soft quasi-proximity by combining fuzzy soft quasi-proximity with fuzzy soft grill and give their properties. Furthermore, they establish the relationship among these fuzzy soft topological structures and their stratifications.

In the contribution by Lur et al. "On the power sequence of a fuzzy interval matrix with max-min operation," authors proposed the notion of max-min algebra of fuzzy interval matrices. They show that the max-min powers of a fuzzy interval matrix either converge or oscillate with a finite period, conditions for limiting behavior of powers of a fuzzy interval matrix are established. Some properties of fuzzy interval matrices in max-min algebra are derived. Necessary and sufficient conditions for the powers of a fuzzy interval matrices in max-min algebra to be nilpotent are proposed as well.

We hope that this special issue would shed light on major developments in the area of real-time image processing systems using fuzzy and rough sets techniques and attract attention by the scientific community to pursue further investigations leading to the rapid implementation of these technologies.

\section{Guest Editors}

\section{Gwanggil Jeon \\ Marco Anisetti \\ Ernesto Damiani \\ Olivier Monga}

Acknowledgements We would like to express our appreciation to all the authors for their informative contributions and the reviewers for their support and constructive critiques in making this special issue possible. 